worten zu politischen, technischen und juristischen Hintergründen der Spähaffäre und der Arbeit der Nachrichtendienste erarbeitet und am 02.09.2013 veröffentlicht.

GI-Präsident Oliver Günther: „Mit der FAQ-Liste liefern Fachleute zu der heiß geführten Diskussion um Ausspähung, Geheimnisverrat und Nachrichtendienste einen neutralen und fundierten Hintergrund. Wir möchten damit zum einen für einen verantwortungsvollen Umgang mit Informationstechnik sensibilisieren, aber auch irrationale Ängste zerstreuen und konkrete Tipps zum Umgang mit persönlichen Daten geben.“

Die FAQ-Liste findet sich im Internet unter der Adresse http:// www.gi.de/themen/ueberwachungsaffaire-2013.html. Die einzelnen Fragen und Antworten sind kommentierbar.

\section{Deutsche IT-Sicherheitswirtschaft gewinnt an Bedeutung}

Angesichts der zunehmenden Digitalisierung der Wirtschaft ist es von zentraler Bedeutung, dass deutsche Unternehmen in den Schutz ihres Know-hows, vor allen auch im Internet, investieren. Die deutsche IT-Sicherheitswirtschaft bietet hier bereits heute viele geeignete Produkte an. Eine Studie im Auftrag des Bundesministeriums für Wirtschaft und Technologie hat den zunehmenden wirtschaftlichen Stellenwert des deutschen IT-Sicherheitsmarkts untersucht.

Die am 11.09.2013 veröffentlichte Studie kommt zu dem Ergebnis, dass die IT-Sicherheitswirtschaft eine der leistungsfähigsten deutschen Zukunftsbranchen ist. Besondere Stärken deutscher ITSicherheitsanbieter liegen in den Bereichen Kryptographie, Smart Cards, PKI-Lösungen, digitaler Signaturen sowie Hochsicherheitslösungen. Angesichts technologischer Innovationen wie dem „Internet der Dinge", der zunehmenden Vernetzung industrieller Leit- und Regelsysteme, Netzwerke und eGovernment-Lösungen wird die IT-Sicherheit auch in Zukunft ein attraktives Geschäftsfeld bleiben. Eine Importquote von nur etwa 20 Prozent in 2012 macht deutlich, dass die Nachfrage nach IT-Sicherheitsprodukten und -dienstleistungen in Deutschland vorwiegend durch die heimische Produktion gedeckt werden und sich die Branche im internationalen Wettbewerb gut behaupten kann. Überdies werden Handlungsfelder für staatliche Aktivitäten zur Förderung der IT-Sicherheitswirtschaft vorgeschlagen. Dazu zählen unter anderem eine stärkere Vernetzung von Forschung und Innovation, die Sicherung des Fachkräftebedarfs sowie die Förderung der Exportfähigkeit kleiner und mittlerer Unternehmen.

Die Studie wurde vom Wirtschaftsforschungsinstitut WifOR der Technischen Universität Darmstadt im Auftrag des BMWi erstellt. Die Studie sowie eine Kurzfassung finden Sie hier: http://www. bmwi.de/DE/Mediathek/publikationen,did=585290.html

\section{Informationsfreiheitsbeauftragte weltweit fordern Stärkung der Transparenz auf nationaler und internationaler Ebene}

Vom 18. bis 20. September 2013 trafen sich Informationsfreiheitsbeauftragte aus 35 Staaten auf der 8 . Internationalen Konferenz der Informationsfreiheitsbeauftragten in Berlin, um mit über 150 Teil- nehmenden aus Politik, Wissenschaft, Verwaltung und Nichtregierungsorganisationen aktuelle Fragen zu Transparenz und Offenheit staatlichen Handelns zu diskutieren.

Die Konferenz wurde ausgerichtet vom Berliner Beauftragten für Datenschutz und Informationsfreiheit Dr. Alexander Dix sowie dem Bundesbeauftragten für den Datenschutz und die Informationsfreiheit Peter Schaar.

Zum Abschluss der 8. Internationalen Konferenz der Informationsfreiheitsbeauftragten in Berlin verabschiedeten die Informationsfreiheitsbeauftragten aus aller Welt eine Entschließung. Das Recht auf Informationszugang müsse gestärkt, die Verpflichtung zur Transparenz staatlichen Handelns erhöht werden. Hierzu teilen die Konferenzausrichter mit:

Peter Schaar: „Unsere Kernbotschaft ist: Alle öffentlichen Stellen auf kommunaler, staatlicher und internationaler Ebene müssen ihr Handeln transparent gestalten. Umfassende Rechte auf Informationszugang sind eine unverzichtbare Voraussetzung für die Beteiligung der Bürgerinnen und Bürger an demokratischen Entscheidungen."

Dr. Alexander Dix: „Auch Geheimdienste dürfen nicht pauschal von Transparenzpflichten ausgenommen bleiben. Effektive Kontrolle von Geheimdiensten setzt ein Mindestmaß an Transparenz voraus. Nur so wird es möglich sein, die unter exzessiver Geheimhaltung stattfindende exzessive Überwachung zu begrenzen."

In der „Berliner Erklärung” unterstützen die Informationsfreiheitsbeauftragten die Anerkennung der Informationsfreiheit als internationales Grundrecht und heben die Bedeutung von Artikel 19 des Internationalen Pakts über bürgerliche und politische Rechte vom 16. Dezember 1966 hervor. Zudem wird empfohlen, dass alle Staaten der Konvention des Europarats über den Zugang zu amtlichen Dokumenten vom 18. Juni 2009 (Tromsø-Konvention) beitreten. Die Bundesrepublik Deutschland gehört zu den Staaten, die der Konvention bisher nicht beigetreten sind.

Die Entschließung finden Sie im Netzunter http://www.datenschutzberlin.de/attachments/977/Resolution_of_the_8_ICIC_Berlin_-_as of_20_September_2013_final.pdf?1379673284

\section{Datenübermittlung in Drittstaaten erfordert Prüfung in zwei Stufen}

Düsseldorfer Kreis am 11./12. September 2013: Beschluss Bei Datenübermittlungen in einen Drittstaat, also einen Staat außerhalb des Europäischen Wirtschaftsraums, sind Datenschutzfragen auf zwei Stufen zu prüfen:

Auf der ersten Stufe ist es erforderlich, dass die Datenübermittlung durch eine Einwilligung der betroffenen Person oder eine Rechtsvorschrift gerechtfertigt ist. Hierbei gelten die allgemeinen Datenschutzvorschriften (z.B. §§ 28 und 32 Bundesdatenschutzgesetz (BDSG)) mit der Besonderheit, dass trotz Vorliegens einer Auftragsdatenverarbeitung die Datenübermittlung nach $\S 4 \mathrm{Abs}$. 1 BDSG zulässig sein muss (vgl. § 3 Abs. 8 BDSG). Bei Auftragsdatenverarbeitung ist der Prüfungsmaßstab in der Regel $\S 28$ Abs. 1 Satz 1 Nr. 2 BDSG, bei sensitiven Daten ist $\S 28$ Abs. 6 ff. BDSG zu beachten.

Auf der zweiten Stufe ist zu prüfen, ob im Ausland ein angemessenes Datenschutzniveau besteht oder die Ausnahmen nach § 4c BDSG vorliegen. 